\title{
LEISHMANIOSE CANINE dANS le RIf (CÔTE MÉdITERRANÉENNE MAROCAINE) : ENQUÊTE SÉRO-ÉPIDÉMIOLOGIQUE
}

\author{
RAMI M.*, ATARHOUCH T.**, SABRI M.*** CADI SOUSSI M.***, BENAZZOU T.* \& DAKKAK A.**
}

Summary: CANINE leishmaniasis in the Rif MOUNTAINS (MOROCCAN MEDITERRANEAN COAST): SERO-EPIDEMIOLOGICAL SURVEY

A sero-epidemiological survey has been conducted in several localities of the province of Nador to investigate canine

leishmaniasis in the North-Eastern slope of the Rif mountains (Mediterranean coast of Morocco). Serum samples collected from 257 dogs were analysed using indirect fluorescent antibody test (IFAT) and enzyme-linked immunosorbent assay (ELISA) to detect anti-Leishmania infantum antibodies. Forty eight (18.7\%) of the screened dogs were IFAT positive and 54 (21.0\%) were ELISA positive; the concordance of the two methods was $96.1 \%$. The prevalence of infection is significantly higher in dogs more than four years of age whereas no significant difference in prevalence of infection was seen between males and females. The frequent symptoms observed in seropositive dogs were the enlargement of lymph nodes (57.4\%), emaciation (51.9\%) and skin involvement (25.9\%). However, $38.9 \%$ of those dogs showed no one of the major symptoms of visceral leishmaniasis. Leishmania isolated from three of the examined dogs was identified as L. infantum MON-1. These results show that the North-Eastern slope of the Rif mountains is one of the most active Mediterranean areas of visceral leishmaniasis and confirm that the dog is the main reservoir of L. infantum.

KEY WORDS : Leishmania infantum, dog, seroprevalence, Mediterranean coast, Morocco.

\section{INTRODUCTION}

D ans le bassin méditerranéen, la leishmaniose viscérale due à $L$. infantum constitue une zoonose dont le chien (Canis familiaris) est le principal réservoir (Bray, 1985; Bettini et Gradoni, 1986). La transmission de l'infection se fait par l'intermédiaire des phlébotomes (Diptera, psychodidae) qui jouent le rôle de vecteur biologique dans le cycle évo-

\footnotetext{
* Département de Biologie, Faculté des Sciences, Université Mohamed V, Rabat, Maroc.

** Département de Parasitologie et Maladies Parasitaires, Institut Agronomique et Vétérinaire Hassan II, BP 6202, Rabat-Instituts, Maroc. *** Laboratoire de Parasitologie, Faculté de Médecine et de Pharmacie, Université Mohamed V, Rabat, Maroc.

Correspondance : Pr. A. Dakkak.

Tél. : 21237776432 - Fax : 21237776432.

E-mail : a.dakkak@iav.ac.ma
}

\section{Résumé :}

Dans le but d'évaluer la situation de la leishmaniose canine dans le foyer situé dans le versant Nord-Est de la chaîne montagneuse du Rif, une enquête séro-épidémiologique a été menée dans différentes localités de la province de Nador (côte méditerranéenne marocaine). Des échantillons de sérum collectés sur 257 chiens ont été analysés par les techniques d'immunofluorescence indirecte (IFI) et d'enzyme-linked immunosorbent assay (ELISA) pour détecter les anticorps antiLeishmania infantum. Les résultats montrent que $48(18,7 \%)$ des chiens examinés sont positifs en IFI et 54 (21,0\%) le sont en ELISA : la concordance des deux méthodes étant de 96, 1 \%. D'autre part, le taux d'infection est significativement plus élevé chez les chiens âgés de plus de quatre ans et ne dépend pas du sexe de l'animal. Les symptômes les plus fréquemment relevés sur les chiens séropositits sont la polyadénite $(57,4 \%)$, l'amyotrophie $(51,9 \%)$ et les lésions cutanéo-muqueuses $(25,9 \%)$. Cependant, $38,9 \%$ de ces mêmes chiens ne présentent pas de symptômes pouvant évoquer la leishmaniose. Des Leishmania isolées sur trois des chiens examinés ont toutes été identifiées comme étant L. infantum MON-1. Ces résultats montrent que le versant Nord-Est des montagnes du Rif est l'un des foyers les plus actifs de leishmaniose viscérale dans le pourtour méditerranéen et confirment le rôle que jove le chien comme principal réservoir de L. infantum.

MOTS CLÉS : Leishmania infantum, chien, séroprévalence, côte méditerranéenne, Maroc.

lutif du parasite. Chez le chien, les leishmanies se localisent dans les cellules du système des phagocytes mononucléés et provoquent des troubles immunopathologiques liés au processus d'auto-immunité et à la formation de complexes immuns (Chang et al., 1985). Malgré la forte incidence chez l'espèce canine, les cas humains recensés restent relativement rares. Cependant, avec l'avènement de la pandémie de SIDA, les foyers situés en Europe méditerranéenne connaissent une recrudescence de la leishmaniose viscérale humaine qui se comporte comme une infection opportuniste chez les sujets immunodéprimés (Bourgeade et al., 1994). Le premier cas de leishmaniose canine au Maroc fut déclaré en 1932 (Jeaume, 1932). Ce n'est, cependant, que durant les deux dernières décennies que cette maladie a suscité un regain d'intérêt et a fait l'objet d'investigations épidémiologiques dans plusieurs régions du Nord du pays (Rioux, 1999). 
Depuis les quinze dernières années, le nombre de cas rapportés par les vétérinaires praticiens du secteur privé et ceux diagnostiqués par le Département de parasitologie et maladies parasitaires de l'Institut agronomique et vétérinaire Hassan II de Rabat ne cesse d'augmenter. Par ailleurs, bien que sporadiques, les cas humains de leishmaniose viscérale recensés, ces dernières années, surtout au front Nord du Maroc, laissaient prévoir l'existence de foyers actifs de leishmaniose canine dans cette partie du pays. Lors d'une enquête épidémiologique effectuée dans la région de Taounate située sur le versant Sud de la chaîne montagneuse du Rif, GuessousIdrissi et al. (1997) ont rapporté chez le chien une séroprévalence moyenne de 16,3\%. Dans une autre étude réalisée dans cinq provinces du Nord du Maroc mais n'incluant pas la région d'étude du présent travail, Nejjar et al. (1998) ont enregistré une séroprévalence de 8,6\%. La présente étude a été réalisée dans la province de Nador qui est l'une des régions du Maroc où un grand nombre de cas de leishmaniose viscérale humaine est enregistré chaque année. En effet, sur une période de 10 ans (1987 et 1996), soixante et un cas ont été recensés, soit plus de 15,25\% du nombre total de cas déclarés dans tout le pays (Ministère de la Santé publique, 1998). L'enquête séro-épidémiologique, basée sur l'utilisation de deux méthodes immuno-sérologiques : l'immunofluorescence indirecte (IFI) et l'enzyme-linked immunosorbent assay (ELISA), a été menée pour évaluer la situation de l'infection canine et contribuer à l'analyse épidémiologique du foyer rifain.

\section{MATÉRIEL ET MÉTHODES}

\section{RÉGION D'ÉTUde}

B ordée par la Méditerranée au Nord, la province de Nador se situe sur le versant Nord-est de la chaine montagneuse du Rif et constitue une zone de transition avec le domaine oriental du Maroc. L'étude a été réalisée dans 10 des 42 communes rurales qui composent cette région (figure 1). La pluviométrie y est assez faible et ne dépasse guère $500 \mathrm{~mm} / \mathrm{an}$; la période humide s'étalant de décembre à avril. La température moyenne annuelle est de $17^{\circ} \mathrm{C}$; la moyenne des maxima du mois le plus chaud (Août) atteint $40^{\circ} \mathrm{C}$ alors que celle du mois le plus froid (Janvier) descend jusqu'à $2^{\circ} \mathrm{C}$. La région est partagée entre deux étages bioclimatiques : un climat semi-aride au niveau du littoral et des zones d'altitude et un climat aride dans les plaines intérieures.

\section{DÉROULEMENT DE L'ENQUÊTE \\ ET ANIMAUX EXAMINÉS}

Dans plusieurs douars (agglomérations rurales de quelques centaines d'habitants) de la zone d'étude, les

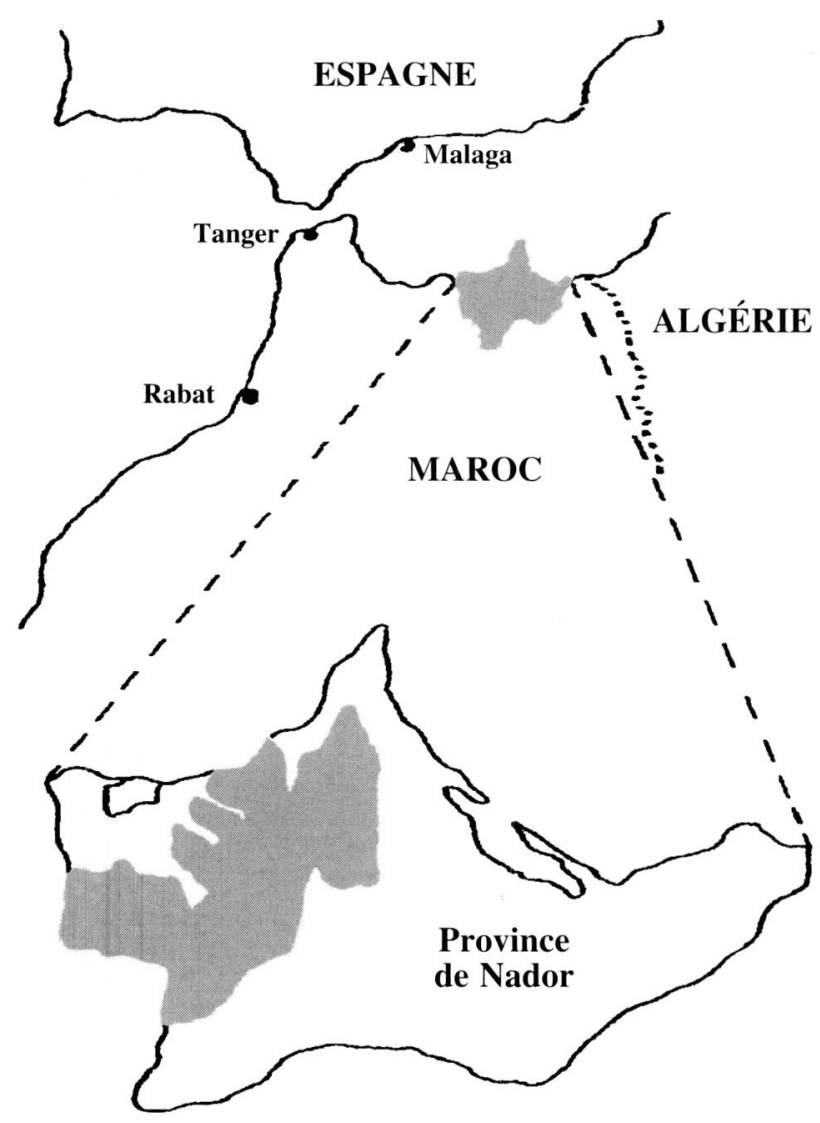

Fig. 1. - région d'étude (틀 = communes concernées par l'enquête.

chiens que les habitants déclarent posséder, bien que vivant en "semi-errance", sont rassemblés, identifiés et font l'objet d'un examen clinique axé sur la recherche des symptômes de la leishmaniose, d'une prise de sang et, lorsque les symptômes relevés laissent suspecter la maladie, d'une ponction du ganglion poplité. Au total, 257 chiens ont ainsi été examinés.

Les sera recueillis à partir des culots plasmatiques sont acheminés au laboratoire et conservés à $-20^{\circ} \mathrm{C}$. Les produits des ponctions ganglionnaires servent à la fois à la confection de frottis colorés au May-GrunwaldGiemsa et à l'isolement du parasite par ensemencement sur un milieu NNN.

\section{SOURCE DE L'ANTIGÈNE}

L'antigène est préparé à partir d'une souche locale de L. infantum isolée sur un des chiens examinés. Il s'agit d'une souche MON-1, dont l'identification a été réalisée par typage enzymatique par nous mêmes (Cadi Soussi et Sabri, 2001) et par le Centre d'Étude sur le Polymorphisme de l'Institut de Recherche et Développement (IRD) de Montpellier (Dr M. Tibayrenc, Communication personnelle).

Pour produire l'antigène en grande quantité, le parasite a été mis en culture dans du RPMI enrichi avec $10 \%$ de sérum fotal de veau. Les promastigotes sont 
récoltés par centrifugation puis lavés dans une solution de $\mathrm{NaCl} 0,15 \mathrm{M}$.

\section{MÉTHODES IMMUNO-SÉROLOGIQUES}

- IFI

Le test IFI a été réalisé selon le protocole décrit par Lanotte et al. (1974). Un volume de $20 \mu \mathrm{l}$ d'une solution d'antigène, contenant $5,10^{6}$ promastigotes $/ \mathrm{ml}$, est déposé dans chaque puits d'une lame à revêtement Téflon. L'antigène est ensuite fixé par immersion dans un bain d'acétone pendant 10 minutes. Après incubation des sera dilués, un anti-sérum de chien marqué à la fluorescéine (Nordic Immunology), dilué à 1/50, est appliqué. Les sera sont d'abord testés à la dilution $1 / 20$ et, dans le cas des sera trouvés positifs à ce titre, la dilution est poussée par progression géométrique de 2 jusqu'à 1/10 240. Les sera dont le titre est supérieur ou égal à 1/160 sont considérés positifs.

\section{- ELISA}

La méthode immunoenzymatique a été réalisée selon le protocole décrit par Okong'Odera et al. (1993). L'antigène utilisé est un extrait soluble obtenu par sonication de promastigotes. Après sensibilisation de la plaque de microtitration par l'antigène dilué dans du tampon carbonate-bicarbonate ( $\mathrm{pH} 9,6)$, à raison d'une concentration protéique de $1 \mu \mathrm{g}$ /puits, les sera, préalablement dilués à $1 / 400$, sont mis en incubation ; chaque échantillon est préparé en duplicata. Un sérum de lapin antiIgG de chien conjugué à la peroxydase (Nordic Immunology), dilué à $1 / 1000$, est ensuite ajouté. La réaction est révélée par l'adjonction d'un substrat chromogène : l'orthophényldiamine. La détermination de la densité optique (DO) se fait à $492 \mathrm{~nm}$ dans un lecteur de plaques ELISA (Titertek Multiscan PLUS).

Le seuil de positivité a été déterminé à partir des DO obtenues chez un lot de 107 chiens cliniquement indemnes, provenant de la région de Rabat reconnue indemne de leishmaniose et dont les examens de frottis ganglionnaires ont tous été négatifs. En supposant que ces valeurs de DO suivent une distribution de type normal, la valeur seuil adoptée est de 0,32. Ce seuil correspond à la moyenne des DO plus 2,6 fois l'écart type et devrait ainsi, inclure $99 \%$ des vrais négatifs.

\section{Analyse statistique}

L'analyse statistique a été réalisée en utilisant le test $\chi^{2}$. Une différence est considérée significative si $\mathrm{P}<0,05$.

\section{RÉSULTATS}

L a répartition de la population canine examinée en fonction des titres d'anticorps obtenus en IFI montre une distribution de type bimodale (figure 2).

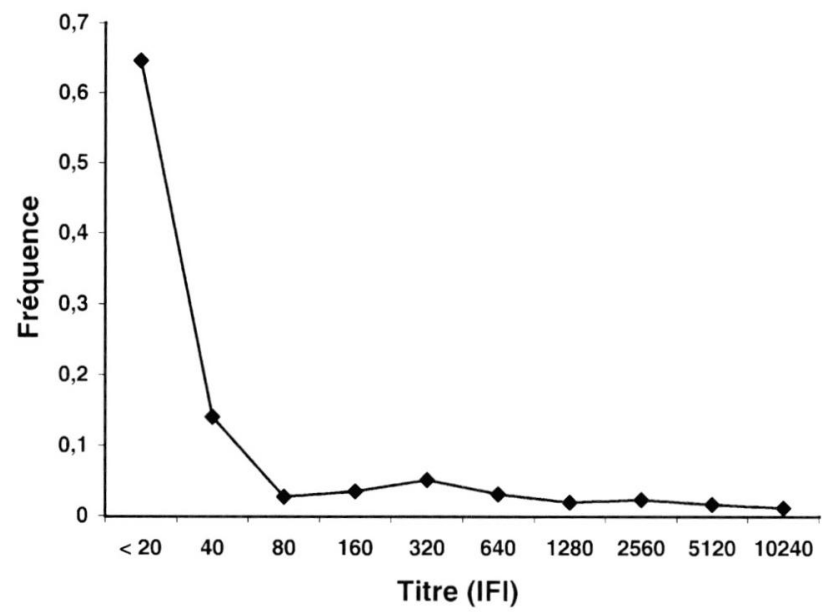

Fig. 2. - Distribution des titres d'anticorps en IFI obtenus chez la population canine examinée.

\begin{tabular}{lccc}
\hline \multicolumn{1}{c}{ Communes } & $\begin{array}{c}\text { Nombre de } \\
\text { chiens examinés }\end{array}$ & $\begin{array}{c}\text { Positifs IFI } \\
\mathbf{( \% )}\end{array}$ & $\begin{array}{c}\text { Positifs } \\
\text { ELISA } \\
\mathbf{( \% )}\end{array}$ \\
\hline Béni Marghanine & 22 & $8(36,4)$ & $9(40,9)$ \\
Tafarsite & 15 & $5(33,3)$ & $6(40,0)$ \\
Ben Taieb & 10 & $3(30,0)$ & $3(30,0)$ \\
Béni Sidel Louta & 11 & $3(27,3)$ & $3(27,3)$ \\
Driouch & 33 & $7(21,2)$ & $8(24,2)$ \\
Azlaf & 19 & $4(21,1)$ & $4(21,1)$ \\
Béni Sidel Jbel & 30 & $6(20,0)$ & $6(20,0)$ \\
Ait Mâit & 35 & $5(14,3)$ & $6(17,4)$ \\
Dar Kabdani & 8 & $1(12,5)$ & $1(12,5)$ \\
Tsaft & 74 & $6(8,1)$ & $8(10,8)$ \\
Total & 257 & $48(18,7)$ & $54(21,0)$ \\
\hline
\end{tabular}

Tableau 1. - Séroprévalence de la leishmaniose canine dans les différentes localités de la région d'étude (région de Nador sur la côte méditerranéenne du Maroc).

Les deux courbes se rencontrent au niveau du titre $1 / 80$.

Sur les 257 chiens examinés, 48 (18,7\%) se sont révélés positifs en IFI et 54 (21,0\%) en ELISA (tableau I). L'enzootie affecte des chiens provenant de toutes les localités concernées par l'enquête avec une prévalence qui dépasse partout $10 \%$. La fréquence la plus élevée atteint 40,9\% (commune de Beni Marghanine). L'étude comparative des titres d'anticorps déterminés en IFI et des DO obtenues en ELISA (figure 3) montre une bonne corrélation avec une concordance de 96,1\% entre les deux tests. Sur 209 sera négatifs en IFI, 201 $(96,2 \%)$ se sont montrés également négatifs en ELISA, alors que 46 de 48 sera positifs en IFI $(95,8 \%)$ ont donné le même résultat en ELISA.

Aucune différence significative n'a pu être mise en évidence entre le taux d'infection des femelles (21,9\%) et celui des mâles (20,3\%). Par contre, la distribution de la séroprévalence en fonction des classes d'âge montre une augmentation significative de la fréquence 


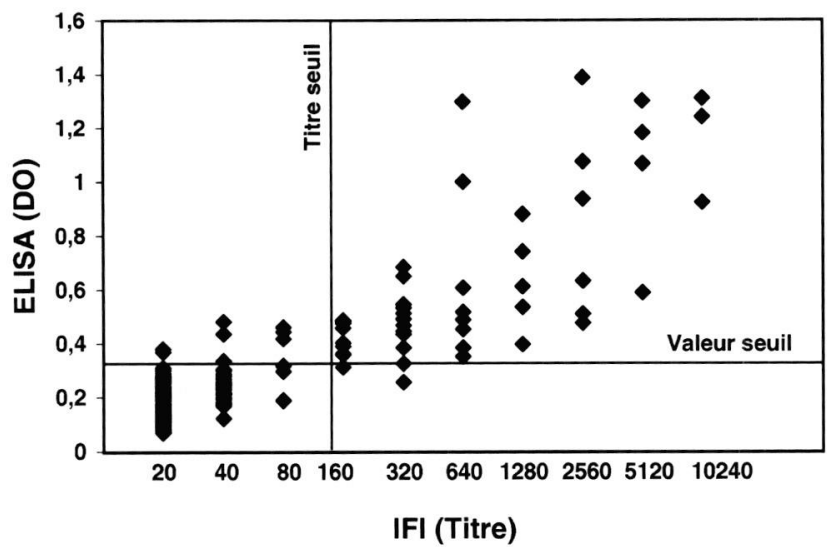

Fig. 3. - Comparaison des résultats des tests IFI et ELSA.

de la maladie à partir de l'âge de quatre ans. En effet, ne dépassant pas 11,1\% chez les chiens âgés de moins de deux ans, la prévalence atteint $28,6 \%$ chez ceux âgés de quatre ans et plus.

Le bilan des examens cliniques révèle que sur les 54 chiens séropositifs en test ELISA, $31(57,4 \%)$ présentent une hypertrophie des ganglions lymphatiques, 28 (51,9 \%) une amyotrophie et 14 (25,9 \%) des signes cutanéo-muqueux (dépilations, dermite furfuracée et ulcérations essentiellement). D'autres symptômes ont été observés mais avec des fréquences plus faibles : onychogriphose $(22,2 \%)$ et kératoconjonctivite ( $4 \%$ ). Enfin, 21 des 54 (38,9\%) chiens séropositifs ne montrent aucun signe clinique apparent de leishmaniose.

\section{DISCUSSION}

L a distribution bimodale des titres d'anticorps en IFI obtenus chez les chiens examinés est caractéristique des zones endémiques. Le même type de distribution a été obtenu dans d'autres régions Méditerranéennes (Lanotte et al., 1974; Abranches et al., 1991). Les deux modes qui expriment successivement les réactions non spécifiques et spécifiques se chevauchent au niveau du titre 1/80. Pour augmenter la spécificité du test IFI, nous avons choisi le titre 1/160 comme seuil de positivité. Selon Lanotte et al. (1974), ce seuil donnerait une meilleure présentation de l'enzootie canine. Au cours des enquêtes séro-épidémiologiques effectuées dans les autres foyers du bassin méditerranéen, différents titres seuil ont été adoptés : 1/40 (Gradoni et al., 1988), 1/80 (Encinas-Grandes et al., 1988 ; Nieto et al., 1992), 1/160 (Dedet et al., 1977 ; Acedo-Sanchez et al., 1996), 1/200 (Ben Saïd et al., 1992 ; Sideris et al., 1996), 1/256 (Ozbel et al., 1995). Cette disparité dans le choix du titre seuil est due aux variations des conditions opératoires et des critères d'interprétation adoptés.
Les résultats des deux tests IFI et ELISA sont concordants dans 96,1\% des cas. Un tel taux de concordance montre la validité de ces deux méthodes pour le diagnostic de la leishmaniose chez le chien et permet de corroborer les taux d'infection enregistrés. Des taux de concordance similaires ont été également rapportés par Semiao-Santos et al. (1995), Sideris et al. (1996) et Scalone et al. (2002). Ainsi, d'exécution relativement facile et d'interprétation précise et objective, le test ELISA convient aux enquêtes épidémiologiques réalisées dans les foyers méditerranéens.

Les taux de séroprévalence enregistrés dans les différentes localités concernées par la présente enquête montrent que le versant Nord-est des montagnes du Rif est le siège d'un foyer hautement enzootique de leishmaniose canine. Avec une fréquence moyenne de $21,0 \%$, il compte parmi les foyers les plus actifs du bassin méditerranéen dont l'Azazga en Algérie avec 37 \% (Belazzoug, 1987), Malaga en Espagne avec 34,6\% (Morillas et al., 1996), Patrimonio en Corse avec 26,5\% (Neogy et al., 1992) et l'île d'Elbe en Italie avec 22,2 \% (Gradoni et al., 1988).

L'identification du zymodème isolé à partir des chiens examinés confirme le rôle du réservoir canin dans le maintien de L. infantum MON-1 aussi bien pour ses congénères que pour l'homme.

Malgré la forte prévalence enregistrée chez le chien, l'incidence annuelle chez l'homme dans la région de Nador ne dépasse pas 0,7 pour 100000 habitants (Ministère de la Santé publique du Royaume du Maroc, 1999). Commune à tous les foyers méditerranéens, cette relative rareté des cas humains peut être due soit aux préférences cynophiles du vecteur, soit à la faible réceptivité de l'homme par rapport au chien (Abranches et al., 1983). La deuxième hypothèse semble être la plus plausible; le nombre élevé de cas positifs au test d'hypersensibilité cutanée à la leishmanine ou celui de la séropositivité, chez les populations humaines qui vivent dans les foyers endémiques (Pampiglione et al., 1975; Acedo-Sanchez et al., 1996 ; Morillas et al., 1996), prouvent que l'homme est en contact étroit avec le parasite sans que la maladie ne soit fréquente. Dans ces conditions, celle-ci se manifeste surtout chez les sujets présentant un déficit majeur de l'immunité cellulaire (Bourgeade et al., 1994). Ceci explique l'augmentation de l'incidence de la leishmaniose viscérale humaine dans les foyers situés en Europe méditerranéenne en parallèle avec le nombre de cas de SIDA (Alvar et al., 1994a ; Tremblay et al., 1996).

En accord avec les résultats de Abranches et al. (1991) et de Sideris et al. (1996), la présente étude montre que le sexe de l'animal n'a aucune influence sur la prévalence de la leishmaniose chez le chien. Par contre, l'âge est un facteur important. En effet, la distribution de la séroprévalence en fonction de l'âge montre une augmentation significative chez les chiens âgés de 
quatre ans ou plus. Des résultats similaires ont été rapportés par Encinas-Grandes et al. (1988), Abranches et al. (1991), Acedo-Sanchez et al. (1996). En effet, la leishmaniose canine étant une maladie chronique dont l'évolution est rarement spontanément régressive, la probabilité pour un chien d'être infecté augmente avec la durée de son exposition au cours des périodes d'activité des phlébotomes et donc avec l'âge.

La présente étude montre que 38,9\% des chiens séropositifs ne présentent pas de signes cliniques de leishmaniose. Ces animaux peuvent être considérés comme des réservoirs du parasite pour leurs congénères et pour l'homme. En effet, Molina et al. (1994) ont constaté que les chiens asymptomatiques ont le même pouvoir infectant pour le vecteur que ceux qui présentent des signes cliniques de la maladie. Abranches et al. (1991) trouvent une fréquence encore plus élevée $(53,8 \%)$. Ces résultats indiquent que chez le chien, la leishmaniose passe par une longue période cliniquement silencieuse d'une part, et montrent l'intérêt de l'utilisation des tests immunologiques aussi bien pour le diagnostic précoce de cette infection que dans les enquêtes épidémiologiques qui la concernent, d'autre part.

En raison de l'efficacité limitée du traitement de la leishmaniose canine, et des taux élevés de rechutes (Lanotte et al., 1979; Alvar et al., 1994b), l'abattage des chiens gravement atteints et le traitement des séropositifs ne présentant pas de symptômes reste le meilleur moyen de lutte contre le réservoir canin. Selon Gradoni et al. (1987) et Dye et al. (1993), l'opération de dépistage et d'élimination des animaux séropositifs doit être effectuée juste avant la saison d'activité des phlébotomes. Cette mesure permettra de détecter les chiens qui ont été infectés pendant la saison précédente après qu'ils aient eu le temps de développer une séroconversion qui ne survient, en général, qu'après un délai de cinq mois après la période de transmission (Vidor et al., 1991). Un tel programme de lutte ne pourrait être entamé que si le foyer d'endémie est bien délimité afin de cibler la zone d'action. Cette stratégie permettra de réduire l'incidence de la maladie sans, pour autant, prétendre en interrompre la transmission. Au Brésil, l'application d'une opération de lutte contre le réservoir canin de L. infantum (chagasi), basée sur l'élimination des chiens séropositifs, a permis de diminuer aussi bien la séroprévalence chez l'espèce canine que l'incidence de la leishmaniose viscérale chez l'homme. Cependant, l'incidence cumulative de la leishmaniose canine n'a été affectée que temporairement (Ashford et al., 1998). Le seul moyen capable de rompre le cycle de transmission du parasite est la vaccination sur laquelle on fonde d'ailleurs, le plus important espoir dans la lutte contre cette zoonose (Hommel et al., 1995 ; Modabber, 1995; Gradoni, 2001).

\section{REMERCIEMENTS}

Te travail a bénéficié d'un financement de la part de la Communauté Européenne (Contrat AVICT92-0010) et d'un appui logistique sur le terrain de la part du Service vétérinaire de la Direction provinciale de l'agriculture de Nador auxquels les auteurs adressent leurs sincères remerciements.

\section{RÉFÉRENCES}

Abranches P., Lopes F.J., Silva F.M.C., Ribeiro M.M.S. \& Pires C.A. Le Kala-Azar au Portugal. III. Résultats d'une enquête sur la leishmaniose canine réalisée dans les environs de Lisbonne. Comparaison des zones urbaines et rurales. Annales de Parasitologie Humaine et Comparée, 1983, 58, 307-315.

Abranches P., Silva-Preira M.C.D., ConceçaO-Silva F.M., SAntos-Gomes G.M. \& Janz J.G. Canine leishmaniasis: Pathological and ecological factors influencing transmission of infection. Journal of Parasitology, 1991, 77, $557-561$

Acedo Sanchez C., Martin Sanchez J., Velez Bernal I.D., Sanchis Martin M.C., Loussini M., Maldonado J.A. \& Morillas MARQuez F. Leishmaniasis eco-epidemiology in Alpujarra region (Granada province, southern Spain). International Journal of Parasitology, 1996, 25, 303-310.

Alvar J. Leishmaniasis and AIDS co-infection. The Spanish example. Parasitology Today, 1994a, 10, 160-163.

Alvar J., Molina R., San Andres M., Tessouro M., Nieto J., Vitutia M., Gonzales F., San Andres M.D., Boggio J., Rodriguez F., Sainz A. \& Escacena C. Canine leishmaniasis: clinical, parasitological and entomological follow-up after chemotherapy. Annals of Tropical Medicine and Paras $i$ tology, 1994b, 88, 371-378.

Ashford D.A., David J.R., Freire M., David R., Sherlock I., Da Conceiça Euliano M., Pedral Sampaio D. \& Badaro R. Studies on control of visceral leishmaniasis: impact of dog control on canine and human visceral leishmaniasis in Jakobina, Bahia, Brazil. American Journal of Tropical Medicine and Hygiene, 1998, 59, 53-57.

Belazzoug S. La leishmaniose canine en Algérie. Maghreb Vétérinaire, 1987, 3, 11-13.

Ben Said M., Jaiem A., Smoorenburg M., Semiao-Santos S.J., Ben Rachid M.S. \& Harith A.E. La leishmaniose canine dans la région d'Enfidha (Tunisie centrale). Estimation de la séro-prévalence par agglutination directe (DAT) et immunofluorescence indirecte (IFAT). Bulletin de la Société de Pathologie Exotique, 1992, 85, 159-163.

Bettini S. \& Gradoni L. Canine leishmaniasis in the Mediterranean area and its implications for human leishmaniasis. Insect Science and its Applications, 1986, 7, 241245.

Bourgeade A., Nosny Y. \& Badiaga S. Aspects cliniques de la leishmaniose viscérale à l'ère de SIDA. Médecine et Maladies Infectieuses, 1994, 24, 566-571. 
BraY R.S. Leishmaniasis in Europe. A brief note. In: Chang K.P. and Bray R.S. (Editors), Leishmaniasis, Amsterdam, Elsevier Science Publishers, 1985, pp. 479-481.

CADI Soussi M. \& SABRI M. Apport de l'identification enzymatique dans les leishmanioses au Maroc : à propos de 33 isolats. Les Cabiers du Médecin, 2001, 4, 47-49.

Chang K.P., Fong D. \& Bray R.S. Biology of Leishmania and leishmaniasis. In: Chang K.P. and Bray R.S. (Editors), Leishmaniasis, Amsterdam, Elsevier Science Publishers, 1985, pp. 1-30.

Dedet J.P., Addadi K. \& Lannuzel B. Epidémiologie des leishmanioses en Algérie. 7. La leishmaniose viscérale dans le foyer de Grande Kabylie. Bulletin de la Société de Patbologie Exotique, 1977, 3, 250-265.

Dye C., Vidor E. \& Dereure J. Serological diagnosis of leishmaniasis: on detecting infection as well as disease. Epidemiology and Infection, 1993, 103, 647-656.

Encinas-Grandes A., Gomez-Bautista M., Martin-Novo M. \& Simon-Martin F. Leishmaniasis in the province of Salamanca, Spain. Prevalence in dog and seasonal dynamics of vectors. Annales de Parasitologie Humaine et Comparée, 1988, 63, 387-397.

GradONi L. An update on antileishmanial vaccine candidates and prospects for canine leishmania vaccine. Veterinary Parasitology, 2001, 100, 87-103.

Gradoni L., Gramiccia M., Mancianti F. \& Pieri S. Studies on canine leishmaniasis control. 2. Effectiveness of control measures against canine leishmaniasis in the isle of Elba, Italy. Transactions of the Royal Society of Tropical Medicine and Hygiene, 1988, 82, 568-571.

Gradoni L., Maroli M., Gramiccia M. \& Mancianti F. Leishmania infantum infection rates in Phlebotomus perniciosus fed on naturally infected dogs under antimonial treatment. Medical and Veterinary Entomology, 1987, 1, 338-342.

Gradoni L., Pizzuti R., Di Matino L., Gramiccia M., Pempinello R., Gaeta G.B., Ferrara M., Scotti S. \& Altieri S. The epidemiology and surveillance of visceral leishmaniasis in the Campania region of Italy. The value of zymodeme typing. Epidemiology and Infection, 1993, 111, 297-306.

Guessous-Idrissi N., Hamdani A., Rhalem A., Riyad M., Sahibi H., Dehbi F., Bichichi M., Essari A. \& Berrag B. Epidemiology of human visceral leishmaniasis in Taounate, a northern province of Morocco. Parasite, 1997, 2, 181-185.

Hommel M., Jaffe C.L., Travi B. \& Milon G. Experimental models for leishmaniasis and testing anti-leishmanial vaccines. Annals of Tropical Medicine and Parasitology, 1995 , 89, 55-73.

JEAUME G. Un cas de leishmaniose naturelle généralisée chez le chien au Maroc. Bulletin de la Société de Pathologie Exotique, 1932, 25, 225-227.

lanotte G., Rioux J.A., Croset H. \& Volhardt Y. Écologie des leishmanioses dans le sud de la France. 7. Dépistage de l'enzootie canine par les méthodes immunosérologiques. Annales de Parasitologie humaine et comparée, 1974, 49, 41-62.

Lanotte G., Rioux J.A., Perieres J. \& Vohlhardt Y. Écologie des leishmanioses dans le sud de la France. 10. Les formes évolutives de la leishmaniose viscérale canine. Elaboration d'une typologie bio-clinique à finalité épidémiologique. Annales de Parasitologie bumaine et comparée, 1979, 54, 277-295.

Ministere de la SANTÉ PubliQue du Maroc. Rapports annuels : État d'avancement des programme de lutte contre les maladies parasitaires (1994-1998). Publication de la Direction de l'Épidémiologie et de la Lutte Contre les Maladies.

Modabbar F. Vaccines against leishmaniasis. Annals of Tropical Medicine and Parasitology, 1995, 89, 83-88.

Molina R., Amela C., Nieto J., San-Andres M., Gonzales F., CAstillo J.A., Lucientes J. \& Alvar J. Infectivity of dogs naturally infected with Leishmania infantum to colonized Phlebotomus perniciosus. Transactions of the Royal Society of Tropical Medicine and bygiene, 1994, 88, 491493.

Morillas F., Sanchez Rabasco F., Ocana J., Martin Sanchez J., Ocana-Wihelmi J., Acedo Sanchez C. \& Sanchiz Marti M.C. Leishmaniasis in the focus of the Axarquia region, southern Spain: a survey of the human, dog and vector. Parasitology Research, 1996, 82, 569-570.

NejJar R., Lemrani M., Malki A., Ibrahimi S., Amarouch H. \& Benslimane A. Canine leishmaniasis due to Leishmania infantum MON-1 in northern Morocco. Parasite, 1998, 5, 325-330.

Neogy A.B., Vouldokis I., Silva O.A., Tselentis Y., Lascombe J., Segalen T., Rzepka D. \& Monjour L. Serodiagnosis and screening of canine visceral leishmaniasis in an endemic area of Corsica: applicability of a direct agglutination test and immunoblot analysis. American Journal of Tropical Medicine and Hygiene, 1992, 47, 772-777.

Nieto C.G., Havarette I., Habela M. \& Hernandez-RodriGUEZ S. Seroprevalence of canine leishmaniasis around Caceres, Spain. Prev. Veterinary and Medicine, 1992, 13, $173-178$.

Okong'Odera E.A., Wamachi A., Kagai J.M., Kurtzhals J.A.L., Githure J.I., Hey A.S., Were J.B.O., Koeche D.K., Mitema E.S. \& Kharazmi A. Field application of an ELISA using redefined Leishmania antigens for the detection of visceral leishmaniasis. Transactions of the Royal Society of Tropical Medicine and Hygiene, 1993, 87, 423-424.

Ozbel Y., Turgay N., Ozensoy S., Ozbilgin A., Alkan M.Z., Ozcel M.A., Jaffe C.L., Schnur L., Oskam L. \& Abranches P. Epidemiology, diagnostic and control of leishmaniasis in the Mediterranean region. Annals of Tropical Medicine and Parasitology, 1995, 89, 89-93.

Pampiglione S., Manson-Bahr P.E.C., La Placa M., Borgatti M.A. \& Musumecr S. Studies on Mediterranean leishmaniasis 3. The leishmanin skin test in kala-azar. Transactions of the Royal Society of Tropical Medicine and Hygiene, 1975, 69, 60-68.

Rıoux J.A. Eco-épidémiologie des leishmanioses au Maroc. Direction de l'Epidémiologie et de la Lutte contre les Maladies. Bulletin Epidémiologique, 1999, 37, 2-9.

Scalone A., De luna R., Oliva G., Baldi L., Satta G., Vesco G., Mignone W., Turilli C., Mondesire R.R., Simpson D., Donoghue A.R., Frank G.R. \& Gradoni L. Evaluation of the 
leishmania recombinant $\mathrm{K} 39$ antigen as a diagnostic marker for canine leishmaniasis and validation of a standardized enzyme-linked immunosorbet assay. Veterinary Parasitology, 2002, 104, 275-285.

Semiao-Santos S.J., El Harith A.E., Ferreira E., Pires C.A., Sousa C. \& Gusmiao R. Evora district as new focus for canine leishmaniasis in Portugal. Parasitology Research, $1995,81,235-239$.

Sideris V., Karagouni E., Papadoupolou G., Garifallou A. \& Dostsica E. Canine visceral leishmaniasis in the Great Athens area, Greece. Parasite, 1996, 3, 125-130.

Trembley M., Olivier M. \& Bernier R. Leishmania and the pathogenesis of HIV infection. Parasitology Today, 1996, 12, 257-261.

Vidor E., Derreure J., Pratlong F., Dubreil N., Bissuel G., Moreau Y. \& Rioux J.A. Le chancre d'inoculation dans la leishmaniose canine à Leishmania infantum. Étude d'une cohorte en région Cévenole. Pratique Médicale et Chirurgicale de l'Animal de Compagnie, 1991, 26, 133-137.

Reçu le 11 mai 2002 Accepté le 15 novembre 2002 